\title{
Counterfeit Resistant F ramework for Single Machine Booking and Grouping in a Production Network Planning Issue
}

\author{
Manikandan Abiraman \\ Department Of Mechanical Engineering, Panimalar Engineering College, Chennai, Tamil Nadu.
}

\begin{abstract}
This paper addresses a generation and outbound dissemination planning issue in which a few occupations must be prepared on a solitary machine for conveyance to clients or to different machines for further handling. We accept that there is an adequate number of vehicles. Likewise, it is expected that the conveyance cost is free of bunch size, yet it is reliant on every excursion. In this paper, we introduce an Artificial Immune System (AIS) for this issue. The goal is to limit the total of the aggregate weighted number of late employ ments and the cluster conveyance costs. A cluster setup time must be included before preparing the principal work in every group. Utilizing computational test, we contrast our technique and a current strategy for the specified iss ue in writing, specifically Simulated Annealing (SA). Computational tests demonstrate the huge change of the AIS over the SA.
\end{abstract}

\section{INTRODUCTION}

Two key operational capacities in an inventory network planning are generation and conveyance operations. In a production network, it is basic to incorporate or all the while consider these two capacities and plan and calendar them together in a planned way to accomplish an ideal operational execution. Established booking issues did not consider circulation and conveyance cost, so considering both the conveyance cost and planning objective in incorporated model is an essential subject. Chen investigated the Integrated Production and Outbound Distribution Scheduling, in particular IPODS, models and arranged these issues into five gatherings. Issues with a target capacity that consider both the machine planning and conveyance are fairly intricate. In any case, they are more useful. In any case, the collection of writing on joined improvement group conveyance issues, particularly with huge size arrangement, is little. Lobby and Potts concentrated the issue of creation booking on a solitary machine under the bunch accessibility presumption (dissemination planning) with a few destinations including the aggregate of stream times, most extreme delay, and the quantity generally occupations. Cluster accessibility supposition implies that every one of the employments shaping a group get to be distinctly accessible for later preparing or dispatch just when the whole clu mp has been handled. They displayed dynamic-programming calculations for limiting the specified goals with conveyance costs when the groups are to be conveyed to a few clients independently. This paper addresses the limiting whole of aggregate weighted number of late occupations and conveyance costs for multi-client in a solitary machine environment and presents an
AIS calculation for comprehending it surprisingly. The base number of late occupations, i.e., is acquired by the polynomial Moore's calculation for the single machine environment. The weighted form of issue, i.e., 1, is hard. For An, a Fully Polynomial Time .Guess Scheme, FPTAS, was given by Sahni. Later, Gens and Levner enhanced it twice. Furthermore, Hallah and Bulfin created Branch and Bound, $\mathrm{B} \& \mathrm{~B}$, technique for this issue considering zero prepared time and non-zero prepared time. Hochbaum and Landy proposed a dynamic programming calculation for the clumping form of the issue, i.e., , in which occupations are prepared in groups which require setup time, and later Brucker and Kovalyov enhanced it. By the by, none of these reviews considered the conveyance costs. Steiner and Zhang tended to the comparable is sue, i.e., booking and clumping is sue conveyance to a client, considering the limiting aggregate of the aggregate weighted number of late employments and conveyance costs on the single machine with cluster setup time; they introduced ideal properties and a pseudopolynomial time DP calculation for the ideal arrangement. Additionally, they introduced a pseudopolynomial DP and a FPTAS for confined instance of multicustomer, where late employments are conveyed independently toward the finish of calendar. As of late, Assarzadegan and Rasti-Barzoki have concentrated the issue of limiting the most extreme lateness, due date task, and conveyance costs on a solitary machine. They introduced two scientific programming models and two metaheuristic calculations, a versatile hereditary calculation, and a parallel-mimicked strengthening calculation, for settling it. A few scientists have connected metaheuristic calculations to take care of planning issue. 
In this exploration, we exhibit an AIS technique for this issue and contrast it and SA and MINLP approaches presented by Mazdeh et al. utilizing computational test is representation of our issues with respect to documentation that Chen presented for these sorts of issues. This documentation implies that there is the single mach ine for handling occupations with group setup time, and adequate vehicle by boundless limit and coordinating conveyance technique for sending the bunches to clients. Coordinating conveyance technique implies that requests are transmitted to every client without the steering issue is the aggregate weighted number of late employments and is the aggregate conveyance costs where and are conveyance costs unit and the quantity of clumps for every client, individually. Chen displayed a vital audit on the writing with respect to coordinated generation and dissemination planning models. Thus, we don't broadly expound.

Whatever remains of this paper is sorted out as takes after: Section 2 contains the issue definition. AIS structure and our proposed calculations are given in area 3 . Area 4 portrays and breaks down the computational outcomes. Furthermore, the last area contains our decisions.

\section{NOTATIONS AND PROBLEM DEFINITION}

\section{2-1. Notations}

Indexes

Job index

Customer index

Parameters:

Nu mber of jobs

Nu mber of customers

Batch setup time for jobs belong to customer

Processing time of job

Due date of job Weight of job

De livery costs for sending batch to customer

Decision variables:

One if job be tardy and zero otherwise

Number of batches for customer Immune system notations:

Nu mber of iteration in local search Population size

Local optimum factor in iteration Mutation rate

Affin ity value of antibody

Control factor of decay

\section{2-2. Problem definition}

There are clients and one producer in which every client orders employments to the maker and is the quantity of occupations. No occupation can be acquired. Every occupation has an imperative coefficient. It is expected that employments require one operation and maker does it by a solitary machine, with preparing time. The due date of occupation is. Occupations are prepared and sent in groups to every client. A clump can contain employ ments just for a similar client. This suspicion is regular in writing for instance observe. We accept that every cluster has a sequence independent-group setup time. There is an adequate number of vehicles, and the conveyance cost is autonomous of bunch size, and it is appeared by for client for each trip. The quantity of groups for client is spoken to by which is a choice variable. The goal is to limit the total of the aggregate weighted number of late employments and conveyance costs. In this way, as specified prior, this is sue can be appeared by 1 .

\section{ARTIFICIAL IMMUNE SYSTEM}

The resistant framework is a data handling and self-learning framework that offers motivation to outline AIS. In the most recent decade, the invulnerable framework has drawn huge consideration as a potential wellspring of motivation for novel ways to deal with take care of complex computational issues. A few scientists utilized the AIS to tackle the booking class issues . In this paper, a metaheuristic calculation in light of AIS interestingly is utilized to limit the aggregate weighted number of late employments and conveyance costs in two-level inventory network. There are a few insusceptible calculations, for example, negative choice calculation, clonal determination calculation, and manufactured resistant systems. In this paper, arrangement method depends on the clonal choice calculation, in which just the most elevated fondness antibodies multiply. So as to comprehend the AIS, some preparatory natural terms are required to be portrayed.

Immune cells: B-cells and T-cells are the two fundamental gatherings of invulnerable cells. These cells help perceive a practically boundless scope of against genic examples.

Antigens (Ag): These are sickness creating components that are partitioned into two sorts of antigens: self and non-self. Non-self-antigens are sickness bringing on components, while selfantigens are innocuous to the body.

Anti bodies (Ab): It is a particle delivered by a Bcell because of an antigen and has the specific property of joining particularly with the antigen, which incited its arrangement.

In the organic procedure, when an antigen contacts with the insusceptible framework, it discharges an arrangement of B-cells, introduce in the immunological memory, with the capacity of recognizing and disposing of the antigens. Those 
B-cells that perceive the antigens with a negligible partiality are decided for cloning and the quantity of clones of a specific cell is characterized by its antigen proclivity. The cells experience substantial hypermutation after the cloning procedure with a specific end goal to attempt to kill the antigen. The cloning and change procedures are rehashed until the antigen is wiped out. At last, the cells with the most astounding proclivity are incorporated into the immunological memory. Hypermutation and receptor altering are two essential attributes of the safe framework. They help in the development of the descendants, as antibodies present in memory reaction must have a higher liking than those in the prior essential reaction. Hypermutation is like the transformation administrator of the hereditary calculation. The distinction lies in the rate of adjustment that relies on upon the antigenic proclivity.

When all is said in done, the antibodies with lower antigenic liking are hypermutated at a higher rate when contrasted with the antibodies with higher antigenic fondness. This marvel is known as receptor altering, which administers the hypermutation. The primary errand of hypermutation is to direct toward nearby ideal, though receptor altering gets away from the neighborhood optima.

In whatever is left of this segment, our AIS properties are presented in detail.

\section{3-1.Fncoding schema}

In the proposed calculation, a counter acting agent incorporates a few qualities, with the end goal that every quality demonstrates the bunch number of every occupation. This encoding plan is appeared in Figure 1. This Figure demonstrates that occupation 1 puts in bunch 5, work 2 puts in clump 2 , and alternate employments in the comparative way. As specified some time recently, proposed recreated tempering calculation to tackle this issue. In their calculation, arrangements are encoded by a grid delineated in Figure 2 where the lines speak to the bunches and the segments speak to the clients. For example, if the component in line 2 and segment 1 is one, the main request of client 1 is appointed to clump 2. Along these lines, for every arrangement, a network is framed with components, though base on our encoding, for every arrangement, a cluster is shaped with components.

\begin{tabular}{|l|l|l|l|l|l|l|l|l|l|l|}
\hline Jobs: & 1 & 2 & 3 & 4 & 5 & 6 & 7 & 8 & 9 & 10 \\
\hline Batches: & 5 & 2 & 2 & 4 & 1 & 3 & 5 & 2 & 1 & 3 \\
\hline
\end{tabular}

\section{3-2. Affinity Calculation}

In the proposed calculation, it is expected to ascertain liking of antibodies. Since the objective is to limit the target work and the proclivity esteem ought to be augmented in the AIS calculations, the less target work esteem is considered as the liking esteem

\section{3-3. The proposed algorithm:}

The main framework of the proposed algorithm is described as follows:

1. Initialization.

2. While (has not met stop criterion) do

3. Local search.

4. Proliferation.

5. Hypermutation.

6. New generation.

7. End.

\section{Initialization}

In this stage, an irregular beginning populace of size is made. For every counter acting agent, the estimation of every quality is resolved haphazardly in the range 1 , in which the estimation of every quality is exceptional. In this paper, with the assistance of starting examination, the extent of populace ( ) is viewed as equivalent to 12 .

\section{Local search}

For every counter acting agent, the accompanying procedure is done circumstances:

One quality is chosen haphazardly. At that point, the estimation of this quality that speaks to the related group is changed to another cluster that incorporates either no employment or alternate occupations of the client of that quality; subsequently, the new arrangement is shaped. At that point, the fondness estimation of the new arrangement is computed. In the event that the blown condition is fulfilled, the counter acting agent will be supplanted by the new arrangement. (1)

Where is the partiality estimation of immune response, is the proclivity estimation of the new arrangement, and is the neighborhood ideal calculate the emphasis. This variable prompts to escape calculation from neighborhood ideal. At to start with, esteem is equivalent to zero; when the best arrangement is not enhanced in three back to back cycles, surprisingly, its esteem will be equivalent to beginning quality. In every emphasis, esteem is diminished base on condition (2) as takes after:

Where is the neighborhood ideal calculate emphasis 1 , is the nearby ideal figure cycle , $\alpha$ is the underlying estimation of neighborhood ideal 
variable, is the quantity of cycle, and is the cycle that the estimation of neighborhood ideal element will be equivalent to introductory incentive surprisingly.

With this condition, the estimation of nearby ideal calculate the last cycle will be equivalent to zero. Subsequently, the assorted qualities in the essential cycles is more prominent than the last emphasess. In this paper, with the assistance of introductory examination, the estimations of and are viewed as equivalent to 0.005 and 80 , individually.

\section{Multiplication}

In this procedure, a few clones are created from every counter acting agent. As in Reisi and Moslehi, the accompanying condition is utilized to compute the quantity of clones that every immune response produces.

Where is the quantity of clones, is the measure of the underlying populace, and is the aggregate likelihood of the counter acting agent. For every counter acting agent, is gotten by isolating its fondness esteem by the whole of all the immunizer affinities.

\section{Hypermutation}

After the expansion organize, the change administrator is performed for every clone. In transformation method, one quality is chosen arbitrarily. At that point, the estimation of this quality that speaks to its cluster is changed to another clump that incorporates either no employment or alternate occupations of the client of that quality. the transformation rate of every clone is figured in light of the accompanying condition:

exp

\section{New populace}

After the hypermutation procedure is done and the liking of the hypermutated arrangements are computed, select the settled number of best immune response for the people to come. In this paper, in light of beginning investigation, is viewed as equivalent to $40 \%$ of PS

\section{Stop basis}

Utilizing computational pre-test, the stop basis is considered as takes after: if the best arrangement is not enhanced after five back to back emphasess or after an aggregate number of 200 cycles, the calculation will be halted

\section{COMPUTATIONAL RESULTS}

In this area, with a specific end goal to assess the execution of AIS, both the little and medium-measure issues are considered. The AIS and SA calculations were coded utilizing Matlab 2009 and keep running on a PC with a $2.93 \mathrm{GHz}$ CPU and a 2.00 GB RAM. The MINLP model was coded in GAMS and illuminated by BONMIN solver, on the grounds that our pre-test demonstrates BONMIN is the most effective solver for tackling the said issue. In little and mediummeasure issues, we have contrasted aftereffects of the proposed calculation and MINLP and SA, individually. The points of interest will be given in the accompanying related subsections.

\section{4-1. Issues with,}

The quantity of employments in little estimated is sues was set $4,7,10$. The quantity of clients for each was characterized by a uniform conveyance in the interim 1,. Preparing times, group setup times, and occupation weights were haphazardly created whole numbers from the uniform dissemination characterized on 1100, 0 $0 . \overline{5}$, and 1100 , individually. In light of the cluster conveyance costs values, we produced two classes of issues, in particular An and $\mathrm{B}$, for every given number of the employ ment. For class An and class $\mathrm{B}$, the interims that the conveyance expenses were produced haphazardly are 0 and $0 \quad 10$,

individually. For every class, we produced three subclasses, to be specific 1, 2, and 3, in light of the due dates values; along these lines, we have six gatherings, in particular A-1, B-1, A-2, B-2, A-3, B-3. For gatherings (A-1, B-1), (A-2, B-2), and (A3 , B-3), the interims that the due dates qualities were produced haphazardly are $0 \quad 0.5,0-$ and 0 5 , separately.

For every employment number in every gathering, 10 issues were created arbitrarily. Subsequently, absolutely $180(3 * 2 * 3 * 10)$ is sues in little measured issues were being created and unraveled. A 300-second time requirement was considered, and if the is sue couldn't be understood with respect to this limitation, then the technique would never again be utilized for that is sue. The aftereffects of the analysis for little measured issues are appeared in Table 1. Segment "Number of the arrangement in which" of Table 1 demonstrates that for all issues, AIS has delivered target work, i.e., add up to cost, less or equivalent to MINLP show. In detail, AIS has delivered target esteem the same as MINLP for $67.22 \%$ of issues and completely a superior outcome for $32.78 \%$ of issues.

In issues with four occupations, both MINLP model and AIS calculation have found the ideal answer for all issues in every gathering, except the normal run time of AIS calculation is littler than the normal run time of MINLP model for every gathering of four jobs. The normal run time of issues with 4 is 0.38 second and 32.26 seconds for AIS and MINLP demonstrate, 
individually. In issues with 7 and 10 occupations, the normal of deviation in A-3 is littler than A-2 and is littler than A-1 in A-2. This implies as the due dates dimin ish the distinction amongst AIS and MINLP, target work increments. What's more, the normal of deviation in $\mathrm{B}-1$ is littler than $\mathrm{A}-1$; in B2 , is littler than A-2; in B-3, is littler than A-3. In this way, as conveyance costs diminish the contrast amongst AIS and MINLP, target work increments.

Tab. 1. The result of experiment for small-sized problems, comparing AIS with MINLP

\begin{tabular}{|l|l|l|l|l|l|l|}
\hline \multicolumn{2}{|c|}{ Delivery } & Due Date Number of the solution in which Ave. of CPU time (s) Costs \\
\hline C classes & Subclass & AIS $<$ MINLP & AIS=MINLP & MINLP<AIS & MINLP & AIS \\
\hline A & 1 & 0 & 10 & 0 & 48.04 & 0.39 \\
4 & 2 & 0 & 10 & 0 & 41.87 & 0.37 \\
& 3 & 0 & 10 & 0 & 7.67 & 0.37 \\
\hline B & 1 & 0 & 10 & 0 & 30.78 & 0.36 \\
& 2 & 0 & 10 & 0 & 50.45 & 0.36 \\
\hline
\end{tabular}

\begin{tabular}{|c|c|c|c|c|c|c|c|}
\hline & C classes & Subclass & AIS $<M I N L P$ & AIS $=$ MINLP & MINLP<AIS & MINLP & AIS \\
\hline \multirow{11}{*}{7} & \multirow{5}{*}{ A } & 3 & 0 & 10 & 0 & 14.76 & 0.41 \\
\hline & & 1 & 8 & 2 & 0 & - & - \\
\hline & & 2 & 6 & 4 & 0 & - & - \\
\hline & & 3 & 0 & 10 & 0 & & \\
\hline & & 1 & 3 & 7 & 0 & & \\
\hline & \multirow[t]{3}{*}{ B } & 2 & 4 & 6 & 0 & - & - \\
\hline & & 3 & 0 & 10 & 0 & - & - \\
\hline & & 1 & 9 & 1 & 0 & & - \\
\hline & \multirow[t]{3}{*}{ A } & 2 & 10 & 0 & 0 & & \\
\hline & & 3 & 1 & 9 & 0 & & \\
\hline & & 1 & 9 & 1 & 0 & & \\
\hline \multirow[t]{2}{*}{10} & B & 2 & 8 & 2 & 0 & & \\
\hline & & 3 & 1 & 9 & 0 & & \\
\hline
\end{tabular}

Cost

*AIS $<$ MINLP implies that AIS has a superior outcome (less aggregate cost) than MINLP

Since a few is sues have not been illuminated inside 300 seconds by GAMS, the st. of CPU time couldn't figured for them

\section{4-2. Issues with}

In this area, we have thought about consequences of our proposed calculation, i.e., AIS, with SA proposed by in medium-sized issues. The quantity of employments in medium-sized issues was set 50,80,110,140. All parameters were produced like the past, however the quantity of issues for every occupation number in every gathering was set 20; consequently, absolutely 480 $(4 * 2 * 3 * 20)$ is sues in medium-sized issues were created. Table 2 demonstrates the aftereffect of the computational test for this is sues.

Tab. 2. The result of experiment in medium-sized problems, comparing AIS with SA

Delivery Costs Due Date Number of the solution in which Ave. of CPU time (s) (\%)

\begin{tabular}{|l|l|l|l|l|l|l|l|l|l|l|l|}
\hline & Classes & & AIS $<$ SA & AIS=SA & SA<AIS & SA & AIS & Avg. & max & Avg. & max \\
\hline 50 & A & 1 & 15 & 0 & 5 & 1.500 & 5.875 & 3.3568 & 18.676 & 0.674 & 5.877 \\
& B & 2 & 20 & 0 & 0 & 1.652 & 4.875 & 13.767 & 58.762 & 0.000 & 0.000 \\
& & 3 & 14 & 6 & 0 & 1.193 & 3.749 & 51.982 & 475.000 & 0.000 & 0.000 \\
& & 1 & 18 & 0 & 2 & 3.548 & 4.639 & 2.738 & 10.109 & 0.041 & 0.768 \\
& & 2 & 16 & 0 & 4 & 2.840 & 5.451 & 1.992 & 10.634 & 0.043 & 0.412 \\
\hline & & 3 & 14 & 6 & 0 & 2.402 & 3.123 & 16.253 & 156.690 & 0.000 & 0.000 \\
\hline 80 & A & 1 & 20 & 0 & 0 & 2.645 & 12.279 & 8.976 & 15.738 & 0 & 0 \\
& B & 2 & 19 & 0 & 1 & 2.564 & 10.788 & 39.657 & 109.876 & 0.089 & 1.772 \\
& & 3 & 20 & 0 & 0 & 1.917 & 5.756 & 35.094 & 215.165 & 0 & 0 \\
& 1 & 20 & 0 & 0 & 4.939 & 12.007 & 6.011 & 28.028 & 0 & 0 \\
& & 2 & 20 & 0 & 0 & 4.428 & 12.196 & 8.645 & 61.892 & 0 & 0 \\
\hline & & 3 & 19 & 1 & 0 & 4.190 & 7.401 & 24.106 & 155.767 & 0 & 0 \\
\hline
\end{tabular}




\begin{tabular}{|l|l|l|l|l|l|l|l|l|l|l|l|}
\hline 110 & $\mathrm{~A}$ & 1 & 20 & 0 & 0 & 4.022 & 22.207 & 14.758 & 42.654 & 0 & 0 \\
& $\mathrm{~B}$ & 2 & 20 & 0 & 0 & 3.810 & 15.892 & 30.030 & 95.338 & 0 & 0 \\
& & 3 & 18 & 2 & 0 & 2.895 & 9.891 & 50.717 & 300.000 & 0 & 0 \\
& & 1 & 20 & 0 & 0 & 7.781 & 22.657 & 10.256 & 44.755 & 0 & 0 \\
& & 2 & 20 & 0 & 0 & 7.302 & 20.461 & 13.377 & 75.304 & 0 & 0 \\
\hline & & 3 & 19 & 1 & 0 & 5.488 & 11.302 & 34.832 & 218.156 & 0 & 0 \\
\hline 140 & $\mathrm{~A}$ & 1 & 20 & 0 & 0 & 5.037 & 34.630 & 14.564 & 72.348 & 0 & 0 \\
& & 2 & 20 & 0 & 0 & 5.250 & 26.626 & 45.090 & 88.867 & 0 & 0 \\
\hline
\end{tabular}

Subclass

Delivery Costs Due Date Nu mber of the solution in which Ave. of CPU time (s) (\%) (\%)

Subclass

\begin{tabular}{|l|l|l|l|l|l|l|l|l|l|l|}
\hline Classes & & AIS<SA & AIS=SA & SA<AIS & SA & AIS & Avg. & max & Avg. & $\max$ \\
\cline { 2 - 10 } & 3 & 19 & 1 & 0 & 3.963 & 17.211 & 108.856 & 595.625 & 0 & 0 \\
\hline B & 1 & 20 & 0 & 0 & 9.941 & 39.466 & 19.169 & 93.312 & 0 & 0 \\
& 2 & 20 & 0 & 0 & 10.794 & 31.236 & 28.379 & 89.023 & 0 & 0 \\
\hline & 3 & 19 & 1 & 0 & 6.759 & 17.689 & 42.434 & 187.731 & 0 & 0 \\
\hline
\end{tabular}

Table 2 demonstrates that the AIS calculation has found a superior arrangement, less target work than SA calculation in $450(93.75 \%)$ issues, and its target capacity is equivalent to SA for $18(3.75 \%)$ issues; consequently, AIS has fathomed $97.5 \%$ of all. problems with less equal total cost with respect to SA; SA has presented a better solution for only $2.5 \%$ of problems. However, the average run time of AIS is larger than SA. Column shows the deviation of SA from AIS when AIS has presented the better result than SA; Column shows the deviation of AIS from SA when SA has presented the better result than AIS. The average deviation of SA from AIS for $93.75 \%$ of problems, for which AIS has presented better result than SA, is $26.04 \%$, while the average deviation of AIS from SA for $2.5 \%$ of problems that SA has presented better result than AIS, is $0.20 \%$. The maximum deviation for SA and AIS is $595.63 \%$ and $5.69 \%$ respectively. These results shows that AIS is more efficient than SA. It is obvious from Table 2 that problems in class B has more average run time, for both SA and AIS, than problems in class A. So, as delivery costs increase, more time was required until the stopping criteria hold. In general, the value for subclass 3 is greater than subclass 2 , and for subclass 2 is greater than subclass 1. Therefore, as due dates increase, the deviation of SA from AIS increases.

\section{CONCLUSION}

This paper presents an AIS algorithm for the scheduling and batching a set of jobs on a single machine with batch setup time for delivery to customers. In order to evaluate the performance of the AIS algorithm, computational tests are used. The computational results show that the proposed AIS framework is more efficient than the MINLP and the SA proposed by . Considering some constraints such as the number of vehicle and capacity for each vehicle, other machine configurations for a manufacturer, such as the parallel machine or flow shop, routing delivery method, instead of directing delivery method, can be suggested for future works. In addition, another function for the total costs, such as total weighted lateness and delivery costs are suggested as well.

\section{REFERENCE}

[1]. Agarwal R, Tiwari M, Mukherjee $\mathrm{S}$. Artificial immune system based approach for solving resource constraint project scheduling problem, The International Journal of Advanced Manufacturing Technology, Vol. 34, Nos. 5-6, (2007), pp. 584-593.

[2]. Gao J. A novel artificial immune system for solving multi objective scheduling problems subject to special process constraint, Computers \& Industrial Engineering, Vol. 58, No. 4, (2010), pp. 602-609.

[3]. Engin O, Döyen A. A new approach to solve hybrid flow shop scheduling problems by artificial immune system, Future Generation Computer Systems, Vol. 20, No. 6, (2004), pp. 1083-1095.

[4]. Chandrasekaran M, et al. Solving job shop scheduling problems using artificial immune system, The International Journal of Advanced Manufacturing Technology, Vol. 31, Nos. 5-6, (2006), pp. 580-593.

[5]. Steiner G, Zhang R. Approximation algorithms for minimizing the total weighted number of late jobs with late deliveries in two-level supply chains, Journal of Scheduling, Vol. 12, No. 6, (2009), pp. 565574

[6]. Dasgupta D, Yu S, Nino F. Recent advances in artificial immune systems: models and applications, Applied Soft 
Computing, Vol. 11, No. 2, (2011), pp. 1574-1587.

[7]. Steiner G, Zhang R. Minimizing the weighted number of late jobs with batch setup times and delivery costs on a single machine, Multiprocessor Scheduling, (2007), pp. 85-98.

[8]. M'Hallah R, Bulfin R. Minimizing the weighted number of tardy jobs on a single machine with release dates, European Journal of Operational Research, Vol. 176, No. 2, (2007), pp. 727-744. 